\title{
Dictionnaire Marguerite Yourcenar, dirigé et préfacé par Bruno BLANCKEMAN
}

\section{Vittorio Fortunati}

\section{OpenEdition}

\section{Journals}

\section{Edizione digitale}

URL: http://journals.openedition.org/studifrancesi/15807

DOI: 10.4000/studifrancesi. 15807

ISSN: 2421-5856

\section{Editore}

Rosenberg \& Sellier

\section{Edizione cartacea}

Data di pubblicazione: 1 décembre 2018

Paginazione: 528

ISSN: 0039-2944

\section{Notizia bibliografica digitale}

Vittorio Fortunati, «Dictionnaire Marguerite Yourcenar, dirigé et préfacé par Bruno BLANCKEMAN», Studi Francesi [Online], 186 (LXII | III) | 2018, online dal 01 janvier 2019, consultato il 06 janvier 2021. URL: http://journals.openedition.org/studifrancesi/15807; DOI: https://doi.org/10.4000/studifrancesi. 15807

Questo documento è stato generato automaticamente il 6 janvier 2021.

\section{(c)}

Studi Francesi è distribuita con Licenza Creative Commons Attribuzione - Non commerciale - Non opere derivate 4.0 Internazionale. 


\title{
Dictionnaire Marguerite Yourcenar, dirigé et préfacé par Bruno
} BLANCKEMAN

\author{
Vittorio Fortunati
}

\section{NOTIZIA}

Dictionnaire Marguerite Yourcenar, dirigé et préfacé par Bruno BLANCKEMAN, Paris, Champion, 2017, $660 \mathrm{pp}$.

1 Alla lunga serie dei dizionari critici pubblicati da Honoré Champion si è aggiunto recentemente, insieme al Dictionnaire Flaubert (a cura di Gisèle Séginger) e al Dictionnaire Jean Giraudoux (a cura di André Job e Sylviane Coyault), quello dedicato a Marguerite Yourcenar, diretto da un importante specialista quale Bruno Blanckeman, docente della Sorbonne Nouvelle. L'opera comprende un totale di trecentoventicinque voci di varia lunghezza, tutte seguite da una bibliografia essenziale ma aggiornata. Tra gli argomenti trattati, oltre alle opere della scrittrice (comprese le interviste e la parte della corrispondenza già pubblicata), troviamo le persone con cui ella fu in rapporto nella sua esistenza (da Brigitte Bardot a Jerry Wilson), gli autori oggetto di saggi critici (da Agrippa d'Aubigné a Virginia Woolf), alcuni personaggi delle sue opere (da Antinoo a Zenone), città, nazioni, movimenti culturali, religioni, generi letterari; vi sono poi un certo numero di voci (da abîme a voyage) dedicate a temi e motivi che hanno un particolare peso nella sua opera e nel suo pensiero. Al Dictionnaire Marguerite Yourcenar hanno lavorato quarantuno studiosi di dieci nazioni diverse, tra le quali la più rappresentata è la Francia (più della metà delle firme), seguita dagli Stati Uniti d'America e dall'Italia: una prova del fatto che gli studi yourcenariani del nostro Paese, tra i più vitali e fecondi, non mancano di visibilità a livello internazionale.

2 La pubblicazione di quest'opera è coincisa, non certo casualmente, con il trentennale della morte della scrittrice e ha risposto a un'esigenza che si avverte da tempo: quella 
di uno strumento di agevole consultazione, che serva da introduzione e, se così si può dire, da vademecum per chi si addentra in un campo di ricerca così ampio e complesso, in cui sono presenti zone ancora poco esplorate. L'utilità del Dictionnaire è, dunque, innegabile e compensa sicuramente le eventuali lacune che si potrebbero riscontrare, realizzando così gli intenti esposti da B. Blanckeman alla fine della sua prefazione: «S'il ne vise pas à l'exhaustivité, le Dictionnaire ainsi conçu nourrit l'ambition de la connaissance critique et le désir d'orienter le lecteur et la lectrice de bonne volonté dans le labyrinthe captivant de l'œuvre» (p.11). Ci auguriamo quindi che non trascorrano molti anni prima che venga data alle stampe una nuova edizione, integrata da altre voci, che dia conto del divenire di uno dei settori più vivaci nell'ambito della ricerca letteraria sul xx secolo. 